\title{
Absorption spectrum in the wings of the potassium second resonance doublet broadened by helium
}

\author{
François Shindo, James F. Babb, Kate Kirby, and Kouichi Yoshino \\ Harvard-Smithsonian Center for Astrophysics, \\ 60 Garden St., Cambridge, MA 02138
}

\begin{abstract}
We have measured the reduced absorption coefficients occurring in the wings of the potassium $4{ }^{2} S-5^{2} P$ doublet lines at $404.414 \mathrm{~nm}$ and at $404.720 \mathrm{~nm}$ broadened by helium gas at pressures of several hundred Torr. At the experimental temperature of $900 \mathrm{~K}$, we have detected a shoulder-like broadening feature on the blue wing of the doublet which is relatively flat between $401.8 \mathrm{~nm}$ and $402.8 \mathrm{~nm}$ and which drops off rapidly for shorter wavelengths, corresponding to absorption from the $\mathrm{X}^{2} \Sigma^{+}$state to the $\mathrm{C}^{2} \Sigma^{+}$state of the $\mathrm{K}-\mathrm{He}$ quasimolecule. The accurate measurements of the line profiles in the present work will sharply constrain future calculations of potential energy surfaces and transition dipole moments correlating to the asymptotes $\mathrm{He}-\mathrm{K}(5 p), \mathrm{He}-\mathrm{K}(5 s)$, and $\mathrm{He}-\mathrm{K}(3 d)$.
\end{abstract}

The broadening of atomic spectral lines through collisions with ambient atoms is of wideranging importance and study of the absorption line shape profiles can reveal much about the absorbers' interactions with the perturbing atoms and with their physical environments. Spectroscopy of the resonance lines of alkali-metal atoms pressure broadened by various elements (including like-species) at temperatures up to $1000 \mathrm{~K}$ has revealed that the far wing absorption profiles possess shapes such as satellite peaks and shoulders indicative of the interactions between absorber and perturber, see, for example, Refs. [1, 2, 3, 4, 5, 6]. Most studies of far wing broadening have centered on the principal series resonance line for an alkali-metal atom in the presence of a ground state inert gas atom. Broadening of the second (and higher) principal lines in these cases has been less frequently studied.

For potassium, there are evidently few experimental studies of the absorption coefficients in the far wings of the second principal doublet $4{ }^{2} S-5^{2} P$ in the presence of helium. Delhoumme et al. measured the absorption coefficients in the presence of neon at $533 \mathrm{~K}$ [7] in an experiment closely related to the theoretical calculations by Masnou-Seeuws [8] of $\mathrm{K}-\mathrm{He}$ 
and of $\mathrm{K}-\mathrm{Ne}$ potential energy curves and transition dipole moments for states correlating to K(4s) and K(5p). Dubourg and Sayer [9] focused their absorption measurements on the red wing in the presence of helium at $548 \mathrm{~K}$. Earlier work using spectrographic methods identified the wavelength at which there is a break off in the satellite band intensity on the blue side of the second doublet [10, 11]. Cantor, Penkin and Shabanova [12] measured the absorption coefficients of the second doublet in the presence of helium at temperatures of around $570-655 \mathrm{~K}$. We report in this letter spectroscopic measurements of the far blue and red wings of the potassium doublet line $4{ }^{2} S-5^{2} P$ broadened by helium at $900 \mathrm{~K}$.

The critical elements in the absorption spectroscopy experiment, illustrated in Figure 1, are a Mach-Zehnder interferometer, a $3 \mathrm{~m}$ Czerny-Turner grating spectrograph (McPherson model 2163) equipped with a CCD camera detector (Andor $1024 \times 256$ pixels with a size of $26 \mu \mathrm{m}$ ), and a gas cell designed for the study of hot and corrosive vapors. This gas cell allows measurements at different buffer gas pressures from 10 Torr to 1000 Torr and temperatures up to $900 \mathrm{~K}$ [13]. The optical path length of the cell is delimited by two $\mathrm{MgO}$ windows separated by a distance of $20 \pm 0.01 \mathrm{~cm}$. A uniform temperature along this path length is maintained by a split tube furnace. For a continuum light source, we use a tungsten halogen lamp $(250 \mathrm{~W}, 24 \mathrm{~V})$ with the beam collimated through an arrangement of optics before entering the interferometer. The gas cell is included in one of the arms of the interferometer. In the other arm, we have placed a stack of windows identical to those contained in the gas cell to compensate for the delay introduced by the optics of the cell. The interferometer is adjusted at optical zero path difference to produce a set of horizontal interference fringes which are focused by a lens on the entrance slit of the spectrometer. Each arm of the interferometer can be blocked by a shutter. Thus, we can measure the spectrum emerging from the gas cell and a reference spectrum emerging from the other arm which serves as a calibration to correct effects due to the variation of the light source intensity with time. The association of the interferometer and the spectrometer is essential to apply the so-called "hook" or anomalous dispersion method which provides a measurement of the atomic number density of the absorbers vaporized in the gas cell.

The hook method uses the change in the refractivity in the vicinity of an atomic spectral line to determine the quantity $N l f$, where $N$ is the atomic number density, $l$ is the optical path length and $f$ is the oscillator strength of the transition producing the spectral line. If $l$ and $f$ are known, then the density $N$ can be determined from the measured value of $N l f$. 
This method and its applications are extensively described by Parkinson [14], Marlow [15], and Huber and Sandeman [16]. As discussed previously, the interferometer is adjusted at optical zero path difference with the gas cell inserted in one arm. In this configuration, we can observe through the spectrometer a set of near horizontal interferences fringes which are spatially distributed along the spectrometer slit. If a dispersive medium exhibiting a resonance line at a certain wavelength $\lambda_{0}$ is introduced into the gas cell, then the variation of the refractive index with wavelength around $\lambda_{0}$ causes a distortion of the fringe profile near the resonance line. By inserting an additional compensation plate into one of the interferometer arms, the fringes are shifted to higher orders, and the deformations appear as hooks on each side of the resonance line. The equations describing the shape of the fringes in the image plane of the spectrometer and extensions of the formalism to the case of hooks formed under the influence of more than one absorption line are given in Refs. [14] and [16]. The vertical position $y_{k}(\lambda)$ of a given fringe of order $k$ (along the spectrometer slit) is a function of the wavelength $\lambda$. For a resonance line doublet, it can be expressed with four free parameters $\alpha, \beta, \delta$ and $\zeta$ as:

$$
y_{k}(\lambda)=\alpha+\beta \lambda+\delta k \lambda+\zeta\left(\frac{A_{1}}{\lambda_{1}-\lambda}+\frac{A_{2}}{\lambda_{2}-\lambda}\right), \quad \text { with } \quad A_{i}=\frac{r_{0} N_{\mathrm{K}} f_{i} l \lambda_{i}}{4 \pi} .
$$

In the configuration of the spectrometer, only the fringes forming on each side of the first resonance doublet around $770 \mathrm{~nm}$ offer a sufficient dispersion and visibility to apply the hook method for the determination of $N_{\mathrm{K}}$, so in our case $\lambda_{1}=766.491 \mathrm{~nm}$ and $\lambda_{2}=769.898 \mathrm{~nm}$. To retrieve the atomic number density $N_{\mathrm{K}}$ of potassium atoms vaporized in the gas cell, we apply a least-squares fit to the shapes of the hooked fringes recorded on the CCD camera, using equation (1). The atomic potassium density $N_{\mathrm{K}}$ is then simply calculated from the ratio $\zeta / \delta$. The wavelength and oscillator strength of each line are taken from Hasegawa et al. [17].

For this experiment, the spectrometer is equipped with a 1200 line/mm plane ruled grating working in first order. A stepper controller can rotate the grating to 116 different fixed angles enabling the spectrometer to cover the wavelength range from $360 \mathrm{~nm}$ to $920 \mathrm{~nm}$. For each angle of the grating position, a spectrum covering about $50 \mathrm{~nm}$ can be recorded by the CCD camera. Each such spectrum was calibrated against available lines from a selection of different standard hollow cathode lamps ( $\mathrm{Na}-\mathrm{Ar}, \mathrm{Ti}-\mathrm{Ne}, \mathrm{Ca}-\mathrm{Ne}$, and $\mathrm{Co}-\mathrm{Ne}$ ). Backlash of the motor driving the rotation of the grating causes an uncertainty of \pm 5 pixels in the 
localization of the calibration lines. In the region of the $404 \mathrm{~nm}$ doublet, this uncertainty corresponds to an error in the wavelength of about $0.04 \mathrm{~nm}$. As an independent check, we measured the forbidden lines $4^{2} S_{1 / 2}-3^{2} D_{3 / 2}$ and $4^{2} S_{1 / 2}-3^{2} D_{5 / 2}$ after calibration and found each differed from the previously reported [18] positions of $464.188 \mathrm{~nm}$ and $464.237 \mathrm{~nm}$, respectively, by only $0.004 \mathrm{~nm}$.

We calculate the absorption coefficient $\tau_{\lambda}$ using Beer's law:

$$
\tau_{\lambda}=-\frac{1}{l} \ln \left(\frac{S_{\lambda}^{\mathrm{K}}}{R_{\lambda}^{\mathrm{K}}} \times \frac{R_{\lambda}^{\mathrm{E}}}{S_{\lambda}^{\mathrm{E}}}\right) .
$$

In equation (2), the quantity $S_{\lambda}^{\mathrm{K}}$ refers to the intensity emerging from the gas cell in presence of potassium vapor and helium. Prior to the vaporization of the potassium in the gas cell, we record a set of backgrounds denoted as $S_{\lambda}^{\mathrm{E}}$ when the gas cell is empty. The ratio between the spectra $R_{\lambda}^{\mathrm{K}}$ and $R_{\lambda}^{\mathrm{E}}$ account for the variation of the light source measured in the reference beam. Assuming that only helium and potassium are involved in the absorption spectrum, in equation (2) we can separate $\tau_{\lambda}$ into two linear terms involving atomic densities $N_{\mathrm{K}}$ and $N_{\mathrm{He}}$, and reduced absorption coefficients:

$$
\tau_{\lambda}=N_{\mathrm{K}} N_{\mathrm{He}} \gamma_{\mathrm{K}-\mathrm{He}}+N_{\mathrm{K}}^{2} \gamma_{\mathrm{K}-\mathrm{K}}
$$

The first term describes the absorption resulting from the collisions between helium and potassium atoms, $\gamma_{\mathrm{K}-\mathrm{He}}$, and the second term describes the pure potassium absorption, $\gamma_{\mathrm{K}-\mathrm{K}}$. The atomic number density of helium atoms is calculated from our measurement of the gas pressure according to the following expression derived from the gas law:

$$
N_{\mathrm{He}}=9.657 \times 10^{18} \frac{P_{\text {Torr }}}{T_{\text {Kelvin }}} \mathrm{cm}^{-3} .
$$

The reduced absorption coefficients can be therefore determined by measuring $\tau_{\lambda}$ at several pressures of helium and/or different densities of potassium.

The stability of the potassium atomic number density in the gas cell is checked before and after the collection of each spectrum. When equilibrium is reached in the gas chamber, the pressure fluctuation of helium lies in a range of $1 \%$ or less around the initial amount, and we observe the same stability for the temperature of the gas cell. For these conditions, the atomic number density of potassium varies within a range of $5 \%$. For a cell temperature of $900 \mathrm{~K}$, we collected several spectra at pressures of helium in the range of 200-900 Torr, and with $N_{\mathrm{K}}$ in the range of $10^{15}-10^{16} \mathrm{~cm}^{-3}$. We have noticed that the continuum varies from one 
spectral measurement to another indicating an irregular decrease of the transmission even when only helium gas is present in the cell. We have established that this phenomenon is related to the gradient of pressure between the gas chamber and the rest of the gas cell even in the absence of potassium vapor in the gas cell. It may be caused by a decrease in the load rate of the springs due to the temperature or the thermal expansion of the windows. In order to obtain absolute absorption coefficients, we have assumed a baseline for our spectra using the experimental spectrum and calculations of Dubourg and Sayer [9]. Their measurements of absorption coefficients shows that the wavelength domain $420-430 \mathrm{~nm}$ is free of $\mathrm{K}-\mathrm{K}$ and $\mathrm{K}-\mathrm{He}$ absorption. Therefore, we can use this wavelength interval in our spectrum to establish our experimental baseline.

For the $\gamma_{\mathrm{K}-\mathrm{He}}$ coefficient profile measured at $900 \mathrm{~K}$ (Fig. 2), we observe a shoulder-like feature on the blue wing of the doublet line with an almost flat intensity of $6.8 \times 10^{-38} \mathrm{~cm}^{5}$ between 401.8 and $402.8 \mathrm{~nm}$ that drops off rapidly for shorter wavelengths. This profile results from an average of several measurements with a standard deviation of $15 \%$. The calculations of Masnou-Seeuws [8] for the $\mathrm{K}-\mathrm{He}$ and the $\mathrm{K}-\mathrm{Ne}$ potentials lead to the prediction of classical satellites on the blue wing of the second resonance doublet. In the case of the $\mathrm{K}-\mathrm{Ne}$ system both theory and experiment exhibit agreement to within $1 \mathrm{~cm}^{-1}$ in the observation of a shoulder-like satellite feature at $403.2 \mathrm{~nm}[7]$. For the K-He system, from the potential energy curves calculated by Masnou-Seuuws, the classical satellite occurs at around $401.5 \mathrm{~nm}$ which differs by $19 \mathrm{~cm}^{-1}$ from our result. Earlier studies, which used photometric analysis of spectrograms, located the peak intensity of the satellite band at $401.76(3) \mathrm{nm}$ [10] and at 401.69(6) $\mathrm{nm}$ [11], but the peak wavelength determination using this method can be affected by the possible presence of background molecular potassium bands. At a wavelength of $402.5 \mathrm{~nm}$, Johnson and Eden [19] measured a signature probably due to $\mathrm{K}_{2}$ in the absorption spectrum of a pure potassium sample. But in our case, considering our experimental densities of potassium and helium, the $\mathrm{K}-\mathrm{He}$ contribution is 500 to 1000 times stronger than the expected contribution from $\mathrm{K}_{2}$. In the present experiment the potassium dimer contribution is ten times weaker than the noise level of $0.4 \%$ in our transmission spectra. Therefore, we can reasonably associate our shoulder-like feature to quasi-molecular absorption from the $\mathrm{X}^{2} \Sigma^{+}$state to the $\mathrm{C}^{2} \Sigma^{+}$state of the $\mathrm{K}-\mathrm{He}$ system. After the completion of the present analysis, we discovered a study published in the Russian literature reporting a similar experiment on the broadening of the $\mathrm{K} 4 \mathrm{~s}-5 \mathrm{p}$ doublet by helium 
and other inert gases in the temperature range of 570-655 K [12]. These authors identify a satellite feature in absorption at $401.85(5) \mathrm{nm}$ in good agreement with our observations at $900 \mathrm{~K}$.

The collisions between potassium and helium give rise to a broadening feature on the blue side of the $4^{2} S-5^{2} P$ second resonance doublet line at around $401.8 \mathrm{~nm}$, in agreement with prior studies [10, 11, 12]. This is consistent with the prediction from previously calculated potential energy curves [8] of a classical satellite feature around this wavelength. Further theoretical calculations of the potential energy curves and transition dipole moments for the molecular states correlating to the asymptotes $\mathrm{He}-\mathrm{K}(5 p), \mathrm{He}-\mathrm{K}(5 s)$, and $\mathrm{He}-\mathrm{K}(3 d)$ would enable more precise predictions of the absorption coefficients. This work is the first result in an extensive study of the absorption arising from $\mathrm{K}-\mathrm{He}$ and $\mathrm{K}-\mathrm{H}_{2}$ gas mixtures. A paper reporting our results on the broadening of the $770 \mathrm{~nm}$ doublet and their application of this work to astrophysical models of the atmospheres of brown dwarfs and certain class of extrasolar planets is currently in preparation [13].

This work was supported in part by NASA under grants NAG5-12751 and NNG06GF06G issued through the Science Mission Directorate, Universe Division, Astronomy and Physics Research and Analysis program. We thank Dr. L. Gardner, Dr. D. Fabricant, and in particular Dr. W. H. Parkinson for valuable advice.

[1] C. L. Chen and A. V. Phelps, Phys. Rev. A 7, 470 (1973).

[2] J. Lorenzen and K. Niemax, Z. Naturforsch. 32a, 853 (1977).

[3] D. Veza, M. Movre, and G. Pichler, J. Phys. B 13, 3605 (1980).

[4] J. Szudy and W. E. Baylis, Phys. Rep. 266, 130 (1996).

[5] M. Shurgalin, W. H. Parkinson, K. Yoshino, C. Schoene, and W. P. Lapatovich, Meas. Sci. Technol. 11, 730 (2000).

[6] C. H. Greene, E. L. Hamilton, H. Crowell, C. Vadla, and K. Niemax, Phys. Rev. Lett. 97, $233002(2006)$.

[7] M. Delhoume, W.-U. L. Brillet, F. Masnou-Seeuws, N. Feautrier, and F. Rostas, J. Phys. B 14, 3857 (1981).

[8] F. Masnou-Seeuws, J. Phys. B 15, 883 (1982). 
[9] I. Dubourg and B. Sayer, J. Phys. B 19, 2291 (1986).

[10] S. Y. Ch'en and R. A. Wilson Jr., Physica 27, 497 (1961).

[11] O. Jefimenko and G. M. Williams, J. Chem. Phys. 42, 207 (1965).

[12] P. Y. Cantor, N. P. Penkin, and L. N. Shabanova, Vestnik Leningrad. Univ. Ser. Fiz. Khim. 3, 80 (1985).

[13] F. Shindo, J. F. Babb, K. Kirby, and K. Yoshino, in preparation.

[14] W. H. Parkinson, Laboratory astrophysics: Atomic spectroscopy, in Spectroscopy of Astrophysical Plasmas, A. Dalgarno and D. Layzer, eds., (Cambridge University Press, New York, 1987), pp. 302-353.

[15] W. C. Marlow, Appl. Opt. 6, 1715 (1967).

[16] M. C. E. Huber and R. J. Sandeman, Rep. Prog. Phys. 49, 397 (1986).

[17] Y. Hasegawa, T. Gejo, K. Tsuji, H. Yamasaki, and S. Shioda, J. Quant. Spectr. Rad. Trans. 45, 97 (1991).

[18] A. R. Striganov and N. S. Sventitskii, Tables of Spectral Lines of Neutral and Ionized Atoms (IFI/Plenum, New York, 1968).

[19] D. E. Johnson and J. G. Eden, J. Opt. Soc. Am. B 2, 721 (1985). 


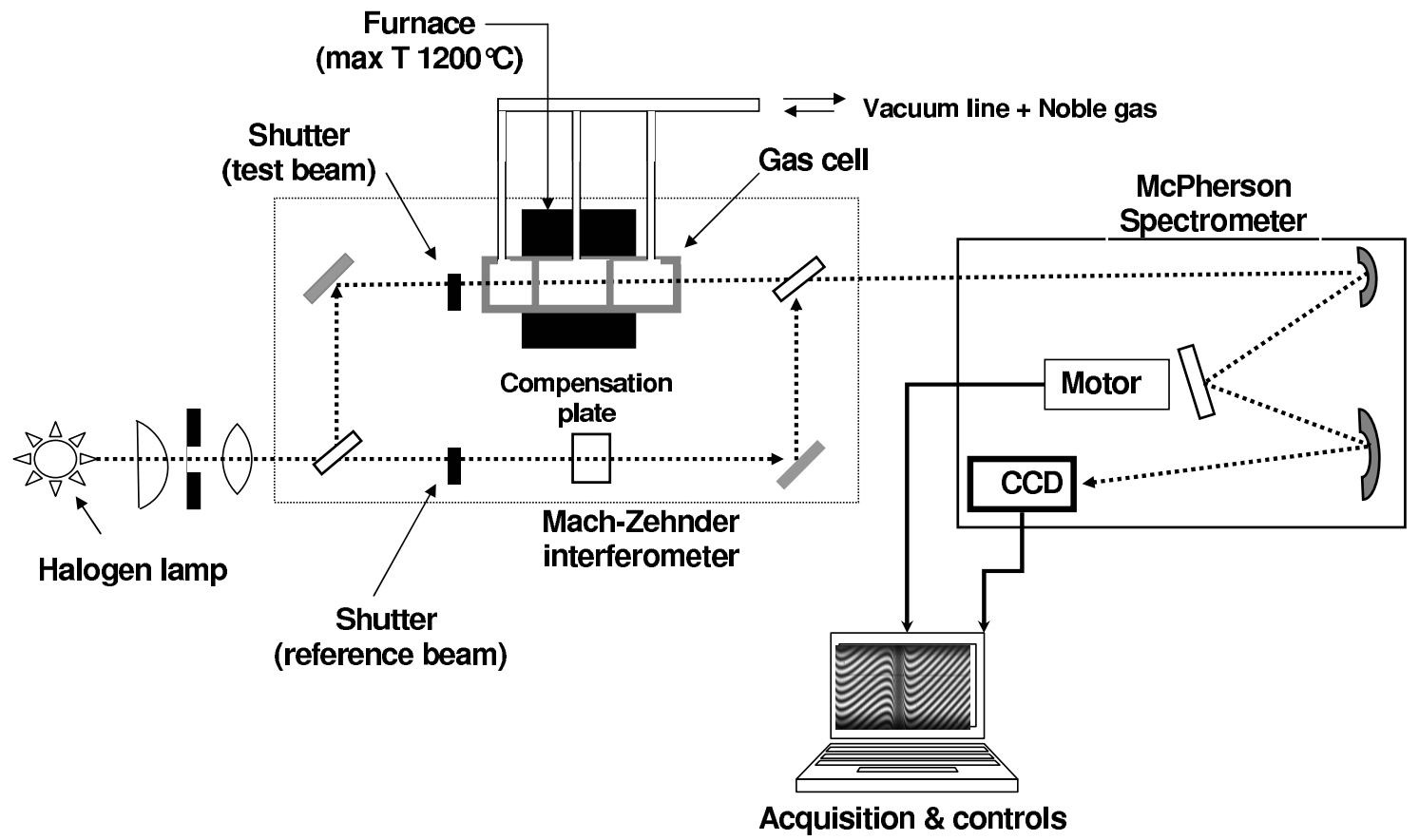

FIG. 1: Experimental set-up as described in the text.

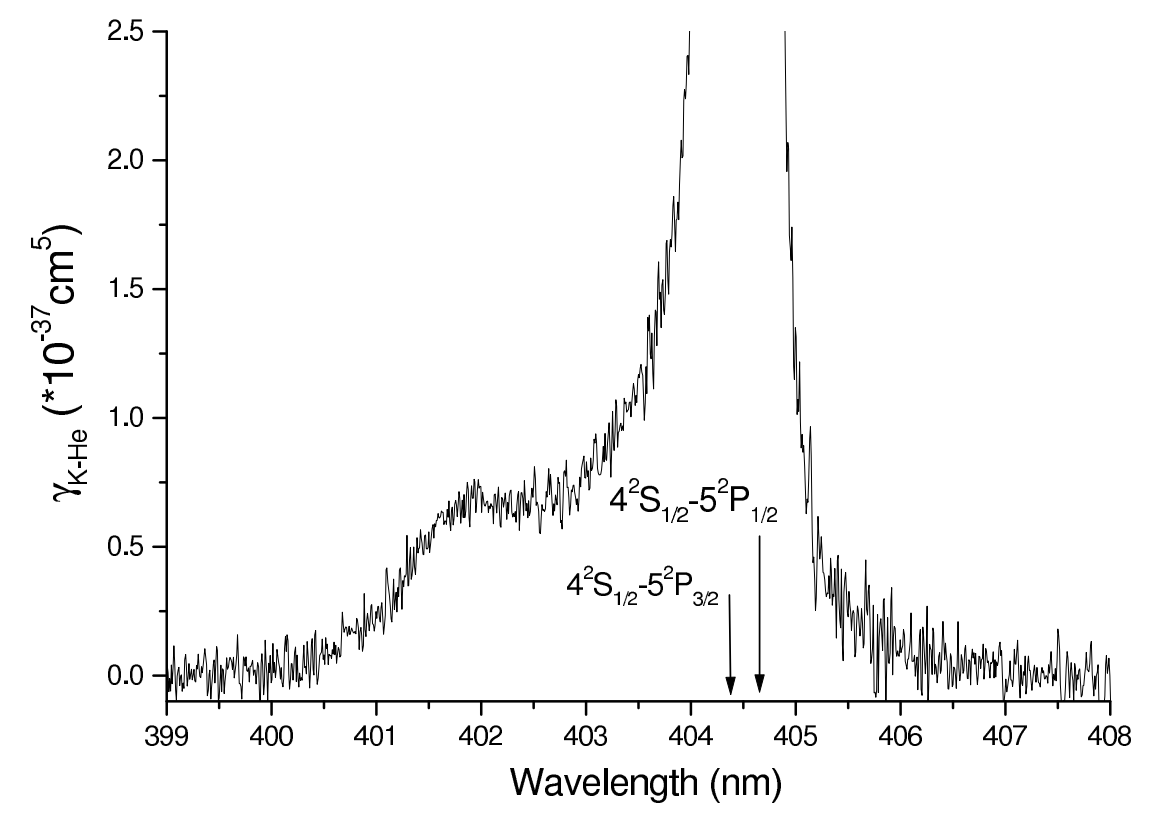

FIG. 2: Reduced absorption coefficient $\gamma_{\mathrm{K}-\mathrm{He}}$ at $900 \mathrm{~K}$. For values less than $1 \times 10^{-37} \mathrm{~cm}^{5}$ the uncertainty is 15 percent and for greater values saturation makes it difficult to assign a consistent uncertainty. 